\title{
Evaluation of Concentrated Bone Marrow-derived Mesenchymal Stem Cells Produced from Two Different Cellular Concentrating Systems
}

\author{
J. M. Brewer ${ }^{1}$, M. B. McCarthy ${ }^{1}$, L. Pauzenburger ${ }^{2}$, M. P. Cote ${ }^{1}$, A. D. Mazzocca ${ }^{1}$ \\ 1 Department of Orthopaedic Surgery, University of Connecticut Medical School, Farmington, Connecticut \\ 2 St. Vincent Shoulder \& Sports Clinic Vienna, Vienna, Austria
}

\author{
CORRESPONDING AUTHOR: \\ Jennifer Brewer \\ University of Connecticut Medical School \\ Department of Orthopaedic Surgery \\ Farmington, CT 06034 \\ Tel: 860-679-6633 \\ Fax: 860-679-6649 \\ Email: jenbrewer830@gmail.com
}

DOI:

10.32098/mltj.03.2019.05

LEVEL OF EVIDENCE: 5

\begin{abstract}
SUMMARY
Background. Concentrated bone marrow-derived mesenchymal stem cells (concentrated bMSCs) are used to improve in-vivo healing, yet in-vivo studies do not describe the cellular components that constitute the concentrated bMSCs. If different concentrating systems create concentrated bMSCs with different cellular components this could affect in-vivo study results. This study sought to compare the cellular components of concentrated bMSCs from two different separating systems.

Methods. Matched bone marrow (BM) and whole blood were commercially obtained. $\mathrm{BM}$ was divided, processed then the concentrated bMSCs were plated and analyzed for cellular concentrates, morphology, triplex, and proliferation assays. The two separation systems used were Angel (system 1) produced from Arthrex, Inc, Naples FL and SmartPrep (system 2) produced from Harvest Technologies, Lakewood, CO. Time points used were 1, 4, 24, 48, 72 and 96 hours. Blinded observers were used to assess morphology of cells at the 24 hour time point.

Results. System 1 had significantly more CFUs $(\mathrm{p}=.0035)$ and more nucleated cells $(\mathrm{p}=.0056)$. Proliferation was significantly increased for system 1 at time points $1,24,48$, 72 , and 96 hours $(\mathrm{p}<.001)$. Cellular viability was significantly increased for system 1 at time points 1,24 and 48 hours $(\mathrm{p}<.05)$. Apoptosis was significantly increased for system 1 at time points 1, 4, 24, 48, 72, 96 hours $(\mathrm{p}<.05)$. Cytotoxicity was significantly increased for system 1 at time points 1, 4, 24, 48, 72, 96 hours ( $\mathrm{p}<.001)$. Morphology of System 1's cells were more consistent of mesenchymal stem cells (MSCs) (Kappa=0.44).

Conclusion. Systems that are used to create concentrated bMSCs create populations of concentrated bMSCs with different cellular properties. Future in-vivo studies involving this cell population should describe the cellular properties of the concentrated bMSCs.
\end{abstract}

\section{KEY WORDS}

Bone Marrow, Mesenchymal Stem Cells, Tissue Healing

\section{BACKGROUND}

Mesenchymal stem cells (MSCs) are able to differentiate into many tissues, including tendon, to augment healing (1- 3). MSCs can be obtained from many types of tissue, including bone marrow (4). Furthermore, these cells can be concentrated. This technique involves harvesting bone marrow (BM) from the patient, using a separation device to concentrate the BM to its constitutional products, then re-inserting the concentrated $\mathrm{BM}$ at the site of injury alone, or with other biological materials to help promote healing
$(2,5)$. There are many studies analyzing the use of concentrated mesenchymal stem cells (concentrated bMSCs) in tissue repair, however, inconsistencies in the methods, specifically in how concentrated bMSCs are obtained can make comparing study results difficult to interpret (6). This study looks to expand on standardizing preparation techniques to create homogenous bMSCs.

The two separation systems used were the Angel system produced from Arthrex, Inc, Naples FL and SmartPrep produced from Harvest Technologies, Lakewood, CO. The 
lab compared varies cellular properties of the two concentrated bMSCs populations created from these machines. The Angel system uses three sensors to accurately separate blood components using cell-specific wavelengths of light to increase cellular yields. Absorption of $470 \mathrm{~nm}$ of light detects plasma and nucleated cells (7). According to the SmartPrep system manual it separates based on a "microprocessor-controlled centrifuge." The lab was not able to obtain further details into the technology, despite numerous attempts to contact the company that makes the device (8). Studies have analyzed how plating densities affect quality and morphology of concentrated bMSC, but these studies only compared different densities using one separation system (9-11). The purpose of this study was to evaluate concentrated bMSCs produced from two separation systems and to evaluate if there are differences between the two concentrated bMSCs products made. The hypothesis is that different separating systems create concentrated bMSCs with similar properties.

\section{MATERIALS AND METHODS}

\section{Bone Marrow Aspiration}

The procedures performed were in accordance with the ethical standards of the journal (12). Native bone marrow of three matched male donors ( $29 \pm 5$ years of age) was purchased (Lonza Inc, Allendale, NJ). According to this company's protocol, $100 \mathrm{mls}$ fresh BM were collected and shipped from the three donors on the same day for next day delivery. Cost of the disposables and assays prohibited us from increasing the amount of bone marrow used. Donors were in good general health according to vital signs and hematology values. Donors had a negative medical history of heart disease, kidney disease, liver disease, cancer, epilepsy, blood or bleeding disorders and tested negative for HIV-1, HIV-2, hepatitis B, and hepatitis C prior to donation. BM (100mls) was obtained from the same company. An aliquot of un-concentrated BM was saved and used as a control in CBC analysis. BM was divided and processed through separation system 1 and separation system 2 to obtain a concentrated product. For all concentrated bone marrow samples, a CBC analysis was done, nucleated cell count (million/ml bone marrow aspirate) and the number of colony forming units (CFU) (CFU/1.0 $\mathrm{ml}$ of bone marrow aspirate) was obtained.

\section{Cell Culture}

Samples were plated in complete alpha minimum essential media (MEM), and contained $10 \%$ fetal bovine serum
(FBS) and $0.1 \%$ penicillin/streptomycin sulfate. Cells were grown in $5 \% \mathrm{CO} 2$, in a $37^{\circ} \mathrm{C}$ incubator. Cells were first counted using the Coulter counter to obtain a nucleated cell count number. An average of three readings was used. Cells were then plated in $100 \mathrm{~mm}$ primaria dishes and colony forming units were counted at the first appearance of colonies between day 7 and 10 in culture.

\section{Proliferation Assay}

The ability of the cells to proliferate was determined by an XTT assay (Roche Diagnostics, Germany). Cells were seeded at a density of 20,000 cells/ cm2 in 24-well plates (Corning Inc., NY, USA) and cultured with complete culture medium at $37^{\circ} \mathrm{C}$ in a $5 \%$ humidified $\mathrm{CO} 2$ incubator. After 48 hours of culture, the cells were incubated with the XTT labeling mixture for 8 hours. At the end of each incubation period, absorbance was measured using an automated plate reader (Bio Tek, Bad Friedrichshall, Germany) at $450 \mathrm{~nm}$ with a reference wavelength of $650 \mathrm{~nm}$.

\section{Triplex Assay}

Cellular viability, apoptosis, and cytotoxicity (inflammation) were measured using an ApoTox-Glo Triplex Assay (Promega Corporation, Madison, WI). The ApoTox-Glo ${ }^{\mathrm{TM}}$ Triplex Assay combines three assay chemistries to assess viability, cytotoxicity caspase activation (showing apoptosis/programed cell death), and cytotoxicity events within a single assay well. The second part of the assay uses a luminogenic caspase- $3 / 7$ substrate, in a reagent optimized for caspase activity, luciferase activity and cell lysis. Cellular viability was measured in relative fluorescence units, apoptosis was measured in relative light units, and cytotoxicity was measured in relative fluorescence units. Time points were 1, 4, 24, 48, 72 and 96 hours. Time chosen was based on the laboratories experience working with concentrated bMSCs in the past. Three different native bone marrows were used to allow for the total number of trials to be 216 . There were 18 trials per time point $(1,4,24,48,72$, and 96 hours), for each individual system.

\section{Cellular Morphology}

Cellular morphology from each system and at each time point was quantified and recorded by three independent researchers in a defined area in each culture plate. It is well documented in the literature that the morphology of MSCs consist of dense, granular areas of individual colonies, which radiate highly and have elongated, flattened spindle-shaped cells with large nuclei forming a dense network of cells that 
strongly adhere to the surface (10,13-15). Using the above criteria, five independent researchers assessed healthy vs. unhealthy colonies obtained from BM. Colonies consisting of six or more cells that are elongated, flattened and firmly attached to the tissue culture plastic, and had processes coming from the cell body were considered healthy colonies. Those cells that had a rounded cell body, were loosely attached to the tissue culture plastic and had no processes coming from the cellular body were considered unhealthy $(10,13-15)$. To avoid bias all observers were given unidentified plates to analyze at the 24 hour time point.

\section{Statistics}

Descriptive statistics were reported using mean and standard deviation to characterize the study conditions. Differences in proliferation, viability, apoptosis, and cytotoxicity between system 1 and system 2 groups at each time point as well as differences across time within each group were examined using mixed linear regression. A p value less than 0.05 was considered statistically significant. Interrater reliability was assessed with the kappa-statistic. Values below 0 were considered poor, 0 to 0.2 slight, 0.21 to 0.4 mild, 0.41 to 0.6 moderate, 0.61 to 0.8 substantial, and 0.81 to 1 almost perfect (16). All analyses were conducted with Stata 15 software (StataCorp. 2017. Stata Statistical Software: Release 15. College Station, TX: StataCorp LLC).

\section{RESULTS}

\section{Cell Analysis}

The findings of the $\mathrm{CBC}$ analysis revealed that there were significantly more WBCs $(\mathrm{p}<.001)$ in system 1 compared to system 2 , but no differences in the number of platelets $(\mathrm{p}=.09)$ or in the RBC count $(\mathrm{p}=.58)$. After cells were counted using a Coulter counter there were significantly more nucleated cells in system $1(\mathrm{p}=.0056)$ and there were significantly more CFUs in system 1 as compared to system $2(\mathrm{p}=.0035)$.

\section{Proliferation and Cellular Assay}

There was significantly more proliferation seen in system 1 as compared to system 2 at time points $1,24,48,72$, and 96 hours $(\mathrm{p}<.001)$ (Figure 1). There was no significant difference between system 1 as compared to system 2 at time point 4 hours $(\mathrm{p}=.065)$. Figure 1 as well demonstrates proliferation significantly increasing over time for system $1(\mathrm{p}<001)$ (Figure 1). Viability was shown to be significantly increased for system 1 compared to system 2 at time points 1 hour $(\mathrm{p}=.001), 24$ hours $(\mathrm{p}<.001)$, and 48 hours $(\mathrm{p}<.001)$ (Figure 2). There was no significant difference found in viability at time point 4 hours $(\mathrm{p}=.805), 72$ hours $(\mathrm{p}=.878), 96$ hours $(\mathrm{p}=.233)$ (Figure 2). Apoptosis was significantly increased

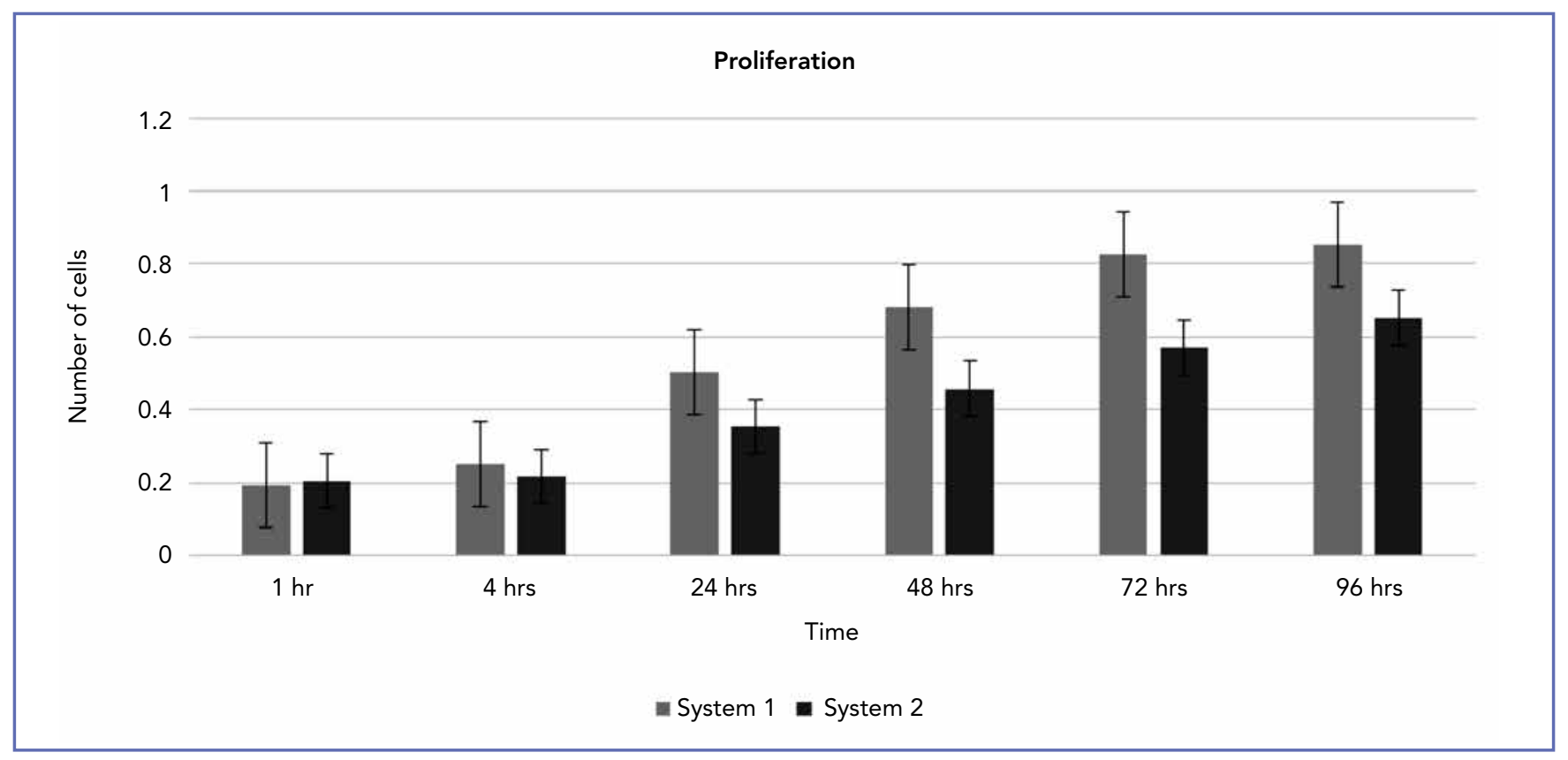

Figure 1. Proliferation (number of cells).

Time points $1,4,24,48,72,96$ hours tested, each time point for each system was $n=36$ for a total $n=216$. Error bars shown. 


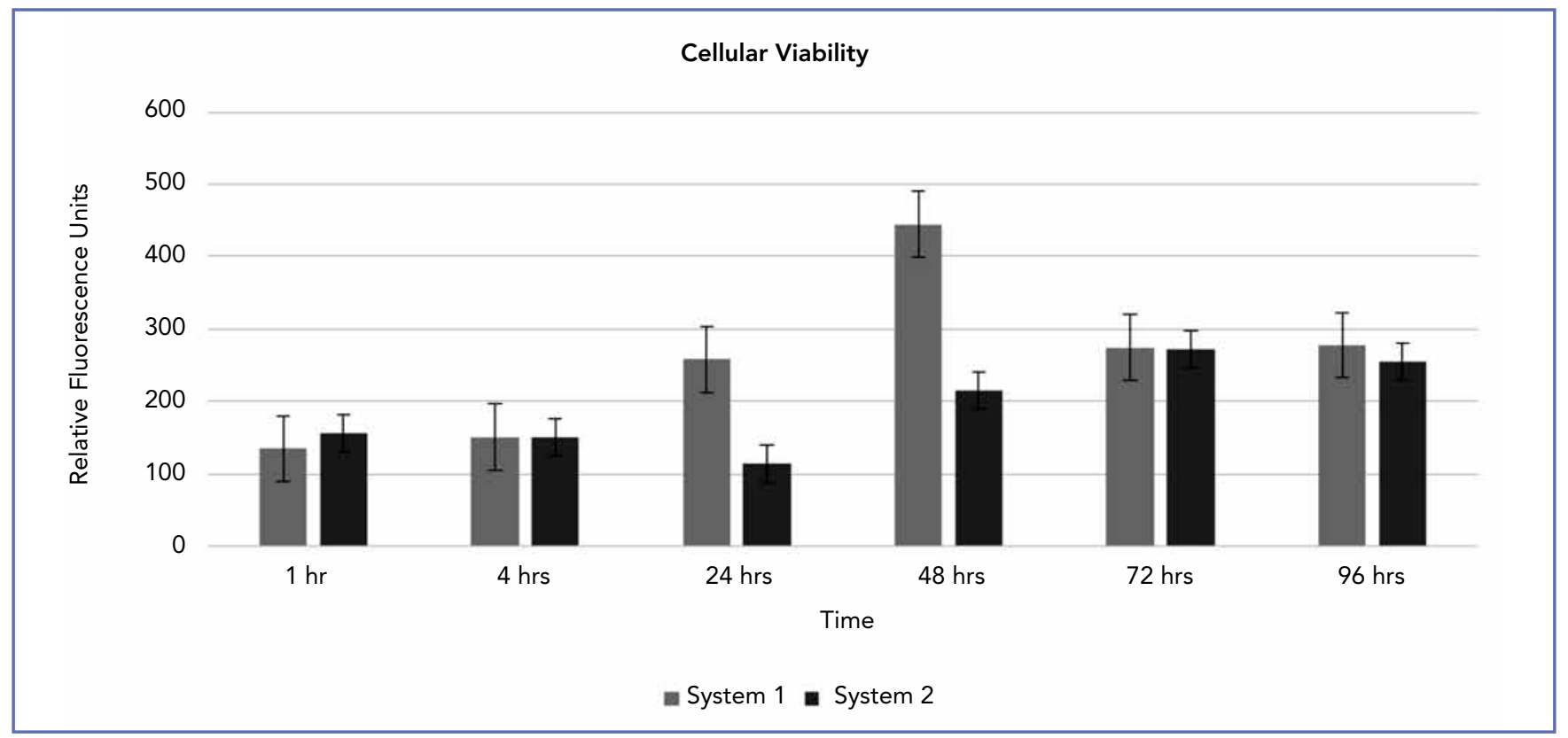

Figure 2. Cellular viability (relative fluorescence units).

Time points 1, 4, 24, 48, 72, 96 hours tested, each time point for each system was $n=36$ for a total $n=216$. Error bars shown.

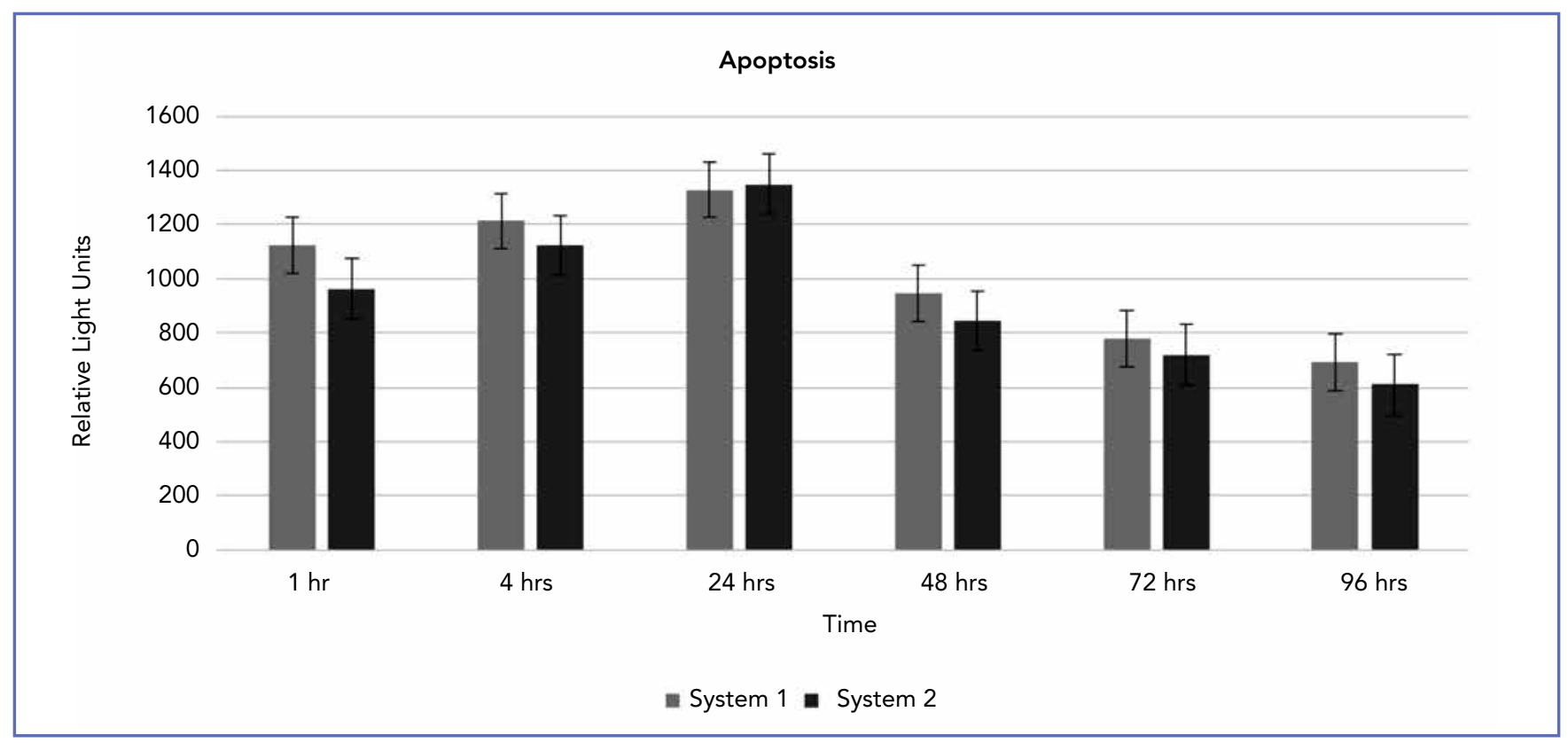

Figure 3. Apoptosis (relative light units)

Time points 1, 4, 24, 48, 72, 96 hours tested, each time point for each system was $n=36$ for a total $n=216$. Error bars shown.

for system 1 as compared to system 2 at time points 1 hour $(\mathrm{p}<.001), 4$ hours $(\mathrm{p}<.001), 24$ hours $(\mathrm{p}<.001), 48$ hours $(\mathrm{p}=.043), 72$ hours $(\mathrm{p}=.047), 96$ hours $(\mathrm{p}=.008)$ (Figure
3). Cytotoxicity was significantly increased for system 1 compared to system 2 at time points 1, 4, 24, 48, 72, and 96 hours $(\mathrm{p}<.001)$ (Figure 4). 


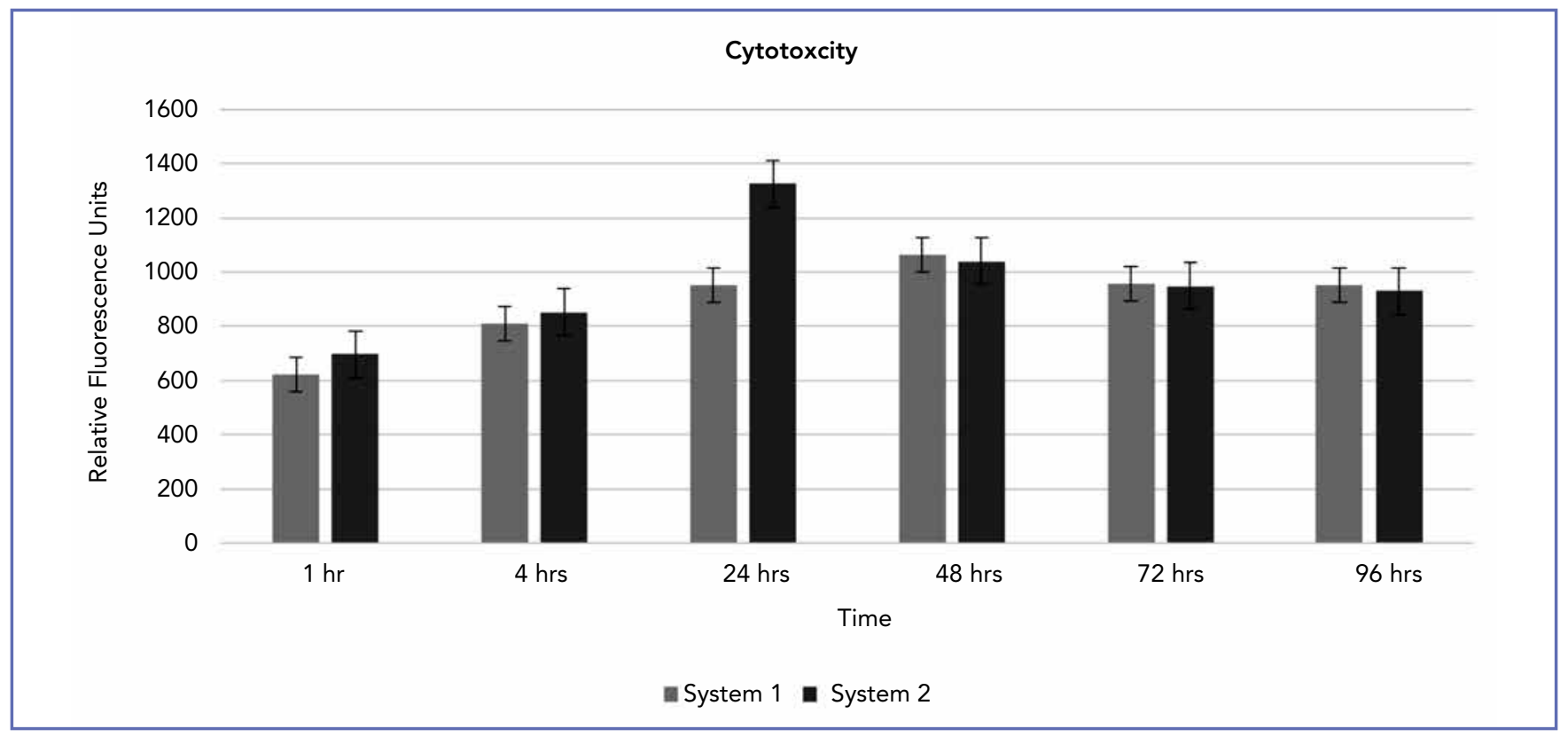

Figure 4. Cytotoxicity (relative fluorescence units)

Time points 1, 4, 24, 48, 72, 96 hours tested, each time point for each system was $n=36$ for a total $n=216$. Error bars shown.

\section{Cellular Morphology}

Out of 5 independent observers all 5 agreed that System 1's cells' morphology looked more like MSCs than those generated by System 2 at the 24-hour time point. Kappa $=0.4400$. Kappa .44 indicates there is moderate interpreter reliability.

\section{DISCUSSION}

Concentrated bMSCs are used in-vivo to help tissue healing. However, most studies involving the in-vivo use of concentrated bMSCs do not include the specific cellular properties of the concentrated cells used, in fact a meta-analysis by Murray et al. found no studies utilizing this technique reported on precise replication protocols nor did the studies provide accurate characterization of the formulation delivered (6). This study sought to explore if different concentrating devices create populations of concentrated bMSCs with different cellular properties. Cellular properties studied include cellular concentrates, cellular morphology, triplex, and proliferation assays. The hypothesis of this study was that different concentrating systems create concentrated bMSCs with similar properties. The results show that the separation systems create groups of cells with different numbers of CFUs and different numbers of nucleated cell counts. In previously published studies it is difficult to compare the amounts of isolated cells because of culture times (until CFU definition), differences in isolation meth- ods, and different media used may result in very diverse outcomes. Hernigou et al. reported positive outcomes in treatment of nonunion and osteonecrosis of the femoral head when concentrated bMSCs were approximately 2500 cells $/ \mathrm{mL}(17,18)$. System 1 produced an average amount of $2561 \mathrm{cells} / \mathrm{mL}$.

The cellular contents of the two concentrated bMSCs populations slightly varied, there was only a significant difference in the amount of WBCs and there was not a significant difference in the amount of platelets or RBCs. As well, there were significant differences between the two systems in the proliferation and triplex assays. It is well described that white blood cells and platelets are helpful in the healing process to help repair tissue and high numbers of red blood cells hinder healing, but the specific concentrates of these cells in concentrated bMSCs to be effective in clinical practice has not been described (19).

Strengths of this study include 18 data points for each time point per separation system, which created a total of 216 data points. This created a stronger statistical power to the study. Detailed cell characteristics of both separation systems were analyzed in this study. As discussed previously, studies have examined how plating densities affect concentrated bMSCs quality and morphology, but these studies only analyzed how plating densities affected one separation system $(9,10)$ and to the lab's knowledge no research has been published 
comparing the concentrated bMSCs produced by two separation systems.

Kappa demonstrated moderate interrater reliability between the students rating the cells. This means that the students were in moderate agreement with each other. There are two Kappa categories higher than moderate, significant and near perfect, so although students were in agreement there was not perfect agreement. It is not understood the underlying mechanism of the difference in morphology between the two systems, but McBeath et al. found MSCs allowed to adhere, flatten, and spread underwent osteogenesis while other cells that were allowed to remain in place and were more round became adipocytes (20). Therefore, it is possible that if the cells adhere to the two systems canisters differently it can cause a differences in the morphology of the concentrated bMSCs produced.

A weakness in this study is that three male BM were used in this study, but the study was not looking at differences between BM, but differences in the products created from separation systems. Cost of the disposables and assays prohibited us from increasing the amount of bone marrow used. As well, the specimens obtained from a commercial supplier may have different characteristics than autograft material harvested at the time of use. Despite numerous attempts to contact system 2's manufacture, the lab was

\section{REFERENCES}

1. Deans RJ, Moseley AB. Mesenchymal stem cells. Exp Hematol. 2000;28(8):875-884.

2. Chaudhury S. Mesenchymal stem cell applications to tendon healing. Muscles Ligaments Tendons J. 2012;2(3):222-229.

3. Via AG, Frizziero A, Oliva F. Biological properties of mesenchymal Stem Cells from different sources. Muscles, Ligaments, Tendons J. 2012;2(3):154-162.

4. Campagnoli C, Roberts IA, Kumar S, Bennett PR, Bellantuono I FN. Identification of mesenchymal stem/progenitor cells in human first-trimester fetal blood, liver, and bone marrow. Blood. 2001;98:2396 -3402.

5. Beitzel K, Solovyova O, Cote MP, et al. The future role of mesenchymal stem cells in the management of shoulder disorders. Arthroscopy. 2013;29(10):1702-1711.

6. Robinson PG, Murray IR, West CC, et al. Reporting of Mesenchymal Stem Cell Preparation Protocols and Composition: A Systematic Review of the Clinical Orthopaedic Literature. Am J Sports Med. 2018:036354651875866.

7. Arthrex. Arthrex Angel System. https://d1psc3qesfsa61.cloudfront.net/pdfs/CfVFVseLk02xlwFEK9V6Pg/ CfVFVseLk02xlwFEK9V6Pg.pdf?Expires=1543336910\&Sig-

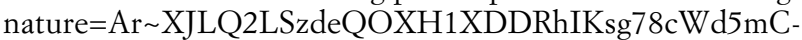
GhY7LxT4XCkl7rAqukKNZh KUnOH2-17znn7RbOaUc0Lp5S3 qgRNv6u kW3D7 oAdZ t97 Waa hE2hQFxL4bl8AVYqYpV-vh5mC. Published 2017. Accessed May 9, 2018. unable to communicate with them. If the lab had greater details into how system 2 worked it may have better understand why the two systems differed in various outcomes. As well, only two separation systems' concentrated bMSCs were compared, there are many more concentrated bMSCs separation devices available. This was an in-vitro study, no in-vivo implications can be derived from the data.

\section{CONCLUSION}

This study found systems that are used to create concentrated bMSCs create populations of concentrated bMSCs with different cellular properties. Future in-vivo studies involving concentrated bMSCs should describe the cellular properties of the cell population created to better understand the clinical significance of concentrated bMSCs in tissue healing.

\section{Conflict of Interest}

This lab receives funding from Arthrex.

\section{Ethics}

The authors declare that this research was conducted following basic ethical aspects as required by the journal (12).

8. Harvest. Operators and Service Manual SmartPReP ${ }^{\circledR} 2$ Centrifuge System. http://www.nupsala.com/wp-content/ uploads/2014/12/Harvest-SmartPReP2-Operators-Manual. pdf. Accessed May 9, 2018.

9. Eslaminejad MB, Nadri S. Murine mesenchymal stem cell isolated and expanded in low and high density culture system: Surface antigen expression and osteogenic culture mineralization. Vitr Cell Dev Biol - Anim. 2009;45(8):451459.

10. Colter DC, Sekiya I, Prockop DJ. Identification of a subpopulation of rapidly self- renewing and multipotential adult stem cells in colonies of human marrow stromal cells. Proc Natl Acad Sci U S A. 2001;3(98):7841-7845.

11. Ikebe C, Suzuki K. Mesenchymal stem cells for regenerative therapy: Optimization of cell preparation protocols. Biomed Res Int. 2014;2014(1).

12. Padulo J, Oliva F, Frizziero A MN. Muscle, Ligaments and Tendons Journal. Basic principles and recommendations in clinical and field Science Research: 2016 Update. Muscles Ligaments Tendons J. 2016;6(1):1-5.

13. Izadpanah R, Trygg C, Patel B, et al. Biologic properties of mesenchymal stem cells derived from bone marrow and adipose tissue. J Cell Biochem. 2006;99(5):1285-1297.

14. Bianco P GRP. Marrow Stromal Stem Cells. J Clin Invest. 2000;105(12):1663-1668. 
15. Bianco P, Riminucci M, Gronthos S RP. Bone marrow stromal stem cells: nature, biology, and potential applications. Stem Cells. 2001;19(3):180-192.

16. Landis JR KG. The measurement of observer agreement for categorical data. Biometrics. 1977;33(1):159-174.

17. Hernigou P, Poignard A, Beaujean F, Rouard H. Percutaneous autologous bone-marrow grafting for nonunions: Influence of the number and concentration of progenitor cells. J Bone Joint Surg Am. 2005;87(7):1430-1437.
18. Hernigou P, Poignard A, Manicom O, Mathieu G, Rouard H. The use of percutaneous autologous bone marrow transplantation in nonunion and avascular necrosis of bone. J Bone Joint Surg Br. 2005;87(B):896-902.

19. Periayah MH, Halim AS, Saad AZM. Mechanism action of platelets and crucial blood coagulation pathways in Hemostasis. Int J Hematol Stem Cell Res. 2017;11(4):319-327.

20. McBeath R, Pirone DM, Nelson CM, Bhadriraju K, Chen CS. Cell shape, cytoskeletal tension, and RhoA regulate stem cell lineage commitment. Dev Cell. 2004;6(4):483-495. 